\title{
Efficacy of TCH/TEC neoadjuvant chemotherapy for the treatment of HER-2-overexpressing breast cancer
}

\author{
WEICAI CHEN, JINSONG HE, SHUFEN SONG, MIN WANG, HUISHENG WU and XIANMING WANG \\ Center for Breast Disease Diagnosis and Treatment, The Second People's Hospital of Shenzhen, \\ Shenzhen, Guangdong 518035, P.R. China
}

Received March 28, 2014; Accepted December 19, 2014

DOI: $10.3892 / 01.2015 .2912$

\begin{abstract}
The aim of the present study was to observe the efficacy of neoadjuvant trastuzumab combined with docetaxel and carboplatin (TCH), and docetaxel, epirubicin and cyclophosphamide (TEC) chemotherapy in human epidermal growth factor receptor-2 (HER-2)-overexpressing breast cancer. The total cohort of 64 cases of HER-2-overexpressing breast cancer patients was divided into two groups according to their treatment preferences: The TCH group, consisting of 39 patients, and the TEC group, consisting of 25 patients. The neoadjuvant chemotherapy was continued for six cycles prior to comparison of the treatment efficacy. The TCG and TEC groups exhibited an overall response rate of 94.9 and $72.0 \%$ (37/39 and 18/25 cases; $\mathrm{P}<0.05)$, respectively, and a pathological complete response ( $\mathrm{pCR}$; defined as the presence of no invasive or in situ residual tumors in the breast) rate of 69.2 and $32.0 \%$ (27/39 and 8/25 cases; $\mathrm{P}<0.05)$, respectively. Furthermore, no significant differences were identified between the two groups of patients in terms of adverse reactions, such as cardiac dysfunction, bone marrow suppression and liver function impairment. In the present study, the treatment of HER-2-overexpressing breast cancer patients with TCH neoadjuvant chemotherapy demonstrated more favorable efficacy and a higher pCR rate when compared with the TEC-treated group.
\end{abstract}

\section{Introduction}

Neoadjuvant chemotherapy is an important treatment strategy for breast cancer and a consensus appears to have been reached with regard to its clinical significance. Previous studies have demonstrated that $20-25 \%$ of breast cancer

Correspondence to: Dr Xianming Wang, Center for Breast Disease Diagnosis and Treatment, The Second People's Hospital of Shenzhen, 3002 Sungang West Road, Shenzhen, Guangdong 518035, P.R. China

E-mail: xmwangpre@163.com

Key words: breast cancer, neoadjuvant, chemotherapy, human epidermal growth factor receptor-2 overexpression patients exhibit human epidermal growth factor receptor-2 (HER-2) overexpression or gene amplification, which results in high biological malignancy and poor prognosis $(1,2)$. HER-2 is a member of the erbB gene family, which is associated with breast cancer (3). HER-2 is expressed during embryonic development and also exhibits an important role in the growth and development of a variety of tissues and organs in adults. HER-2 overexpression in breast cancer most frequently occurs as a result of gene amplification. The overexpression of HER-2 produces a malignant phenotype, which leads to cell proliferation and subsequently a more aggressive tumor phenotype, and thus, patients with HER-2 overexpressing breast cancer exhibit a poor prognosis (4). Furthermore, HER-2 status has been reported to be an independent risk factor for the relapse of the disease (5). Despite marked improvements in the prognosis of HER-2 positive breast cancers, which have been observed due to the widespread use of HER-2-specific therapies, such as trastuzumab, patients continue to exhibit recurrences and disease progression (6). Pathological complete response (pCR) may be used to discriminate between patients with favorable and unfavorable outcomes in response to treatment (7). pCR is a suitable surrogate end point for patients with HER-2-positive tumors (8), and the prognostic impact of pCR is highest in HER2-positive tumors. Trastuzumab-based preoperative neoadjuvant therapy with trastuzumab plus docetaxel and carboplatin ( $\mathrm{TCH}$ ) has previously been reported to achieve a promising efficacy, with a good $\mathrm{pCR}$ rate and favorable tolerability in stages II or III in Her-2-positive breast cancer (9). Docetaxel in combination with epirubicin and cyclophosphamide (TAC) has also been used as preoperative systemic therapy for locally advanced breast cancer (10-12). However, the treatment efficiency of TCH and TAC neoadjuvant chemotherapies on Her-2 positive breast cancer has not been comparatively studied. Data from previous studies have demonstrated that among breast cancer driven by HER-2 overexpression, a treatment plan that combines neoadjuvant chemotherapy with trastuzumab may produce a significantly higher pCR rate compared with chemotherapy alone $(13,14)$. In the present study, HER-2-overexpressing patients were administered six cycles of trastuzumab combined with docetaxel and carboplatin (TCH), or docetaxel, epirubicin and cyclophosphamide (TEC) as neo adjuvant chemotherapy prior to surgery. The outcomes were then analyzed in order to compare the clinical efficacy of each treatment. 


\section{Patients and methods}

General clinical data. Between May 2009 and October 2012, the Center for Breast Disease Diagnosis and Treatment (Shenzhen, China) enrolled 64 female patients who had developed breast cancer as a result of HER-2 overexpression into the present study. The patients were all examined by performing a core needle biopsy (CNB) and all diagnoses of breast cancer were established for the first time. The age of the patients varied between 23 and 60 years, with a median age of 38 years. According to the international tumor-node-metastasis (TNM) staging system (15), the cohort included three cases of stage IIB, 21 cases of stage IIIA and 40 cases of stage IIIB breast cancer (Table I). The patients were divided into two groups, according to their treatment preferences, the TCH group $(n=39)$ and the TEC group $(n=25)$. All patients had undergone ultrasonic, X-ray and bone mineral density scans to confirm the absence of distant metastases prior to commencing chemotherapy, and no patients had undergone any treatment, such as chemotherapy, radiotherapy, traditional Chinese medicine or endocrine therapy, prior to admission to the Center for Breast Disease Diagnosis and Treatment. All human studies were approved by the ethics committee of the Second People's Hospital of Shenzhen (Shenzhen, Guangdong, China), and were thus performed in accordance with the ethical standards of the 1964 Declaration of Helsinki and its later amendments. In addition, all participants provided informed consent prior to their inclusion in the present study.

\section{Pathological examination and scoring systems}

Pathological examination. CNB specimens were fixed in $10 \%$ neutral formaldehyde solution and paraffin-embedded, then three $4-\mu \mathrm{m}$ thick sections were cut from each paraffin wax block. The sections were subjected to neuropeptide substance P immunohistochemical analysis (16), with all reagents purchased from Maixin Biotechnology (Fuzhou, China), and routine immunohistochemistry staining was conducted by performing a 2,4-dinitrobenzene chromogenic reaction (17). Furthermore, phosphate-buffered saline was used as the negative control instead of primary monoclonal mouse anti-human HER-2 (dilution 1:1; cat. no. 4B5; Roche, Indianapolis, IN, USA), monoclonal mouse anti-human ER (cat. no. MAB-0062; 1:200; Fuzhou Maixin Biotech Co., Ltd., Fuzhou, China) and monoclonal mouse anti-human PR (cat. no. MAB-0231; 1:200; Fuzhou Maixin Biotech Co., Ltd.) antibodies, and tissue sections positive for estrogen receptor (ER), progesterone receptor (PR) and HER-2 were used as the positive controls.

Scoring systems. The ER and PR staining results were classified using a semi-quantitative histochemical scoring system previously established by Allred et al (18); however, the present study only considered the intensity of the stained cells. The intensity score ranged from 0 to 3 as follows: 0 , no staining (-); 1 , weak staining (+); 2 , intermediate staining (++); and 3 , intense staining $(+++)$, with positive expression noted as $\mathrm{ER}(+)$ or $\mathrm{PR}(+)$ and a lack of positive staining noted as ER(-) or PR(-). Furthermore, Her-2/neu protein expression levels were scored on a scale of 0 to 3 , according to a system previously utilized by Almasri and Al Hamad (19). The cellular staining was scored as follows: (i) Negative (-),
Table I. General clinical data of all breast cancer patients $(n=64)$.

\begin{tabular}{lrr}
\hline & \multicolumn{2}{c}{ Cases } \\
\cline { 2 - 3 } Parameter & $\mathrm{n}$ & $\%$ \\
\hline TNM stage & 3 & \\
IIB & 21 & 32.8 \\
IIIA & 40 & 62.5 \\
IIIB & & \\
Pathological type & 44 & 68.8 \\
Invasive ductal carcinoma & 20 & 31.2 \\
Infiltrating lobular carcinoma & & \\
Receptor expression & 23 & 35.9 \\
ER(+)/PR(+) & 13 & 20.3 \\
ER(+)/PR(-) & 12 & 18.8 \\
ER(-)/PR(+) & 16 & 25.0 \\
ER(-)/PR(-) & & \\
Axillary lymph node metastasis & 7 & 10.9 \\
$\quad$ Negative & 57 & 89.1 \\
Positive & & \\
\hline
\end{tabular}

TNM, tumor-node-metastasis; ER, estrogen receptor; PR, progesterone receptor.

no membrane staining or staining in $<10 \%$ of the tumor cells; (ii) weak positive (+), weak focal membrane staining in $>10 \%$ of the tumor cells; (iii) intermediate $(++)$, weak to moderate complete membrane staining in $>10 \%$ of the tumor cells; and (iv) strongly positive (+++), weak to moderate cytoplasmic reactivity and intense membrane staining in $>10 \%$ of the tumor cells. In addition, HER-2(-) or HER-2(+) represented a lack of HER-2 overexpression and HER-2(+++) indicated HER-2 overexpression. HER-2(++) results were double-checked by performing fluorescence in situ hybridization (FISH), using FISH(+) as HER-2 overexpression and FISH(-) as no HER-2 overexpression (20).

\section{Treatment strategy}

TCH regimen. On day one (the first day of each chemotherapy cycle), the TCH regimen was administered as $75 \mathrm{mg} / \mathrm{m}^{2}$ docetaxel intravenously, with a carboplatin area under the curve of six. Trastuzumab was intravenously infused once a week at $4 \mathrm{mg} / \mathrm{kg}$ for the first week, $2 \mathrm{mg} / \mathrm{kg}$ for the following 16 weeks (17 weeks total) and $6 \mathrm{mg} / \mathrm{kg}$ every three weeks for one year after surgery. Cardiac function was monitored at baseline, and at three, six and nine months after day one.

$T E C$ regimen. Docetaxel was administered via an intravenous drip at a dose of $75 \mathrm{mg} / \mathrm{m}^{2}$ on day one. On day two, $80 \mathrm{mg} / \mathrm{m}^{2}$ epirubicin was intravenously administered along with $500 \mathrm{mg} / \mathrm{m}^{2}$ cyclophosphamide.

For the two regimens, one chemotherapy cycle lasted for 21 days and six cycles were conducted in total. Two weeks after the completion of the neoadjuvant chemotherapy regimes, modified radical mastectomy or breast conserving therapy were performed. 
Table II. Comparison of OR and pCR rates of the two treatment groups.

\begin{tabular}{lcccr}
\hline & \multicolumn{2}{c}{ Regimen, \% (n) } & \\
\cline { 2 - 4 } Efficacy & TCH $(\mathrm{n}=39)$ & TEC $(\mathrm{n}=25)$ & $\chi^{2}$ & P-value \\
\hline OR & $94.9(37)$ & $72.0(18)$ & 4.838 & 0.028 \\
pCR & $69.2(27)$ & $32.0(8)$ & 7.085 & 0.008 \\
\hline
\end{tabular}

OR, overall response; pCR, pathological complete response; TCH, docetaxel, carboplatin and trastuzumab; TEC, docetaxel, epirubicin and cyclophosphamide.

Evaluation of treatment efficacy. For the patients in the two treatment groups, magnetic resonance imaging was employed prior to and following the treatment to determine the size of the tumor and to evaluate the efficacy of each chemotherapy regime.

According to the uniform criteria established by the World Health Organization, the efficacy of the treatment strategies was classified as follows: Clinical complete response (cCR), disappearance of all tumors/lesions upon clinical examination; partial response $(\mathrm{PR}), \geq 50 \%$ decrease in tumor size; stable disease (SD), $<50 \%$ decrease in tumor size or $<25 \%$ increase in tumor size; and progressive disease (PD), $\geq 25 \%$ increase in tumor size or development of new lesions (21). Additionally, overall response (OR) was calculated as CR plus PR, and pCR was defined as the presence of no invasive cancerous cells at the in situ tumor region of the surgical specimen, with no concurrent metastasis in the axillary lymph nodes.

Statistical analysis. SPSS software (version 17.0; SPSS Inc., Chicago, IL, USA) was used to perform the $\chi^{2}$ test on the data and $\mathrm{P}<0.05$ was considered to indicate a statistically significant difference.

\section{Results}

Groups. Her-2/neu was shown to be overexpressed in all patients [+++: 45; ++: 14; + : 5, 19 cases (Her-2++/+) were double-checked by FISH and were demonstrated to be Her-2/neu overexpression FISH(+)]. The cohort of 64 patients was randomly separated into two groups: The TCH group, consisting of 39 patients, and the TEC group, consisting of 25 patients. No significant differences in age, tumor staging or pathological characteristics were identified between the two groups.

Curative efficacy. The TCH and TEC groups exhibited OR rates of 94.9 and $72.0 \%$, respectively $(\mathrm{P}<0.05)$, and $\mathrm{pCR}$ rates of 69.2 and $32.0 \%$, respectively $(\mathrm{P}<0.05)$ (Table II). These results indicated that $\mathrm{TCH}$ chemotherapy was more efficacious than TEC chemotherapy for the treatment of HER-2 overexpressing breast cancer.

Adverse reactions. No treatment-associated mortality or cardiac insufficiency occurred in any of the patients enrolled in the present study. Eight patients in the TEC group exhibited symptoms, including shivering and fever, upon the initial infusion of trastuzumab; however, these adverse effects were remitted once the remainder of the treatment was applied.
All patients experienced myelosuppression to varying degrees, and the ratio of grade III-IV neutropenia in the TCH and TEC groups was $25.6 \%$ (10/39 cases) and $28.0 \%$ (7/25 cases), respectively, demonstrating no statistical significance $(\mathrm{P}=0.928)$. Treatment proceeded when the leukocyte level was recovered following granulocyte-colony stimulating factor (G-CSF) therapy.

The patients in the two groups demonstrated different levels of gastrointestinal reactions, such as nausea, vomiting and diarrhea, which were relieved following antiemetic treatment and fluid supplementation. In addition, patients in the two groups only exhibited oral ulcers, rash, peripheral neurotoxicity and hepatic dysfunction to a mild extent, which were tolerable after expectant treatments.

\section{Discussion}

Neoadjuvant chemotherapy, with its broad and ever-increasing number of applications, is now substantially important in integrated therapies for breast cancer. Previous studies have revealed that neoadjuvant chemotherapy, compared with traditional adjuvant chemotherapy, may eliminate early subclinical disseminated lesions, and reduce the TNM stage of primary lesions and regional lymph nodes $(22,23)$. Neoadjuvant chemotherapy may additionally provide information on the chemotherapeutic sensitivity of the tumor and the prognosis of patients, establishing it as particularly effective in the treatment of local terminal breast cancer. Neoadjuvant chemotherapy has become one of the standard therapeutic regimens for the treatment of late clinical stage breast cancer (24) and was therefore used in the present study, in which $95.3 \%$ (61/64 cases) of patients were in the late clinical stages, including 21 stage IIIA patients and 40 stage IIIB patients.

Previous clinical studies have indicated that breast cancer patients with HER-2 overexpression typically demonstrate a poor prognosis and a high degree of malignancy in tumorous biological behavior $(25,26)$. In recent years, the increasing clinical application of trastuzumab has significantly improved the disease-free and overall survival (OS) rate of patients (27). Although trastuzumab is predominantly employed post-operatively or in the treatment of metastatic HER-2-overexpressing breast cancer, clinicians worldwide have hypothesized that a trastuzumab-associated chemotherapeutic scheme may enhance the pCR of HER-2-overexpressing breast cancer patients by a large margin $(28,29)$.

In the BCIRG006 clinical trial (29), a TCH regimen of trastuzumab combined with docetaxel and carboplatin was 
designed. The three-year median follow-up period indicated that the efficacy of this joint chemotherapy regimen of trastuzumab and non-anthracyclines was similar to the regimen of doxorubicin and cyclophosphamide with sequential docetaxel and trastuzumab (AC-TH), but with fewer cardiac toxicity reactions. Additional studies indicated that trastuzumab and docetaxel exhibit a synergistic effect when administered together, markedly improving the response rate of HER-2-overexpressing breast cancer by increasing the time to progression, as well as the median survival time (30-33). Furthermore, another study reported that the efficacy of neoadjuvant chemotherapy combined with trastuzumab was significantly greater than that of chemotherapy alone in the treatment of HER-2-overexpressing patients (pCR, 31.7 vs. 15.7\%) (34). Currently, pCR is the most important indicator for assessing the efficacy of neoadjuvant chemotherapy regimens $(35,36)$. The results of the present study indicated that the efficacy of treating 39 HER-2-overexpressing breast cancer patients with the TEC regimen was most favorable, demonstrating $94.9 \%$ OS and $69.2 \%$ pCR rates after six cycles of observation. This high $\mathrm{pCR}$ rate may be explained by the previous medical history of enrolled patients, the timely and effective treatment of side-effects and completion of the six-cycle treatment by all patients.

Major adverse reactions are caused by the use of trastuzumab and chemotherapeutic agents. Of the reactions caused by trastuzumab, fever appears to be the most common response, predominantly occurring during the first infusion as opposed to after the administration of medication. Remission of fever may be achieved via the application of conventional antipyretic analgesics and anti-allergy medicines. However, cardiac dysfunction is the most severe type of adverse reaction, and may manifest as dyspnea, pulmonary edema, peripheral edema and cardiac dilatation (37). In a previous study, the rate of symptomatic heart failure was 4-6\% when trastuzumab was used alone, however, the rate was significantly higher $(27 \%)$ when combined with anthracyclines (38).

Adverse reactions caused by the administration of chemotherapeutic agents include myelosuppression to various degrees, gastrointestinal reactions, oral ulcers and alopecia (39); however, in the present study, none of the 39 patients in the TCH group experienced severe toxic side-effects or cardiac insufficiency. The underlying explanation for this may be that the enrolled subjects had no history of heart disease, the observation of $a \geq 50 \%$ left ventricular ejection fraction upon ultrasonography or the application of an anthracycline-free neoadjuvant chemotherapy scheme. Despite the lack of severe side-effects, patients in the two groups did experience grade III-IV neutropenia, with treatment commencing following G-CSF treatment and a recovery of leukocyte levels. Other adverse reactions were comparatively mild, and all were tolerated following the administration of expectant and supporting treatments.

In conclusion, the neoadjuvant chemotherapy regimen of trastuzumab combined with docetaxel and carboplatin exhibited significant efficacy, tolerable adverse reactions and a high pCR rate in the HER-2-overexpressing breast cancer patients. Thus, the TCH regimen should be applied, particularly for the treatment of late clinical stage breast carcinoma.

\section{Acknowledgements}

This study was supported by the Shenzhen Science and Technology Plan Project (grant no. 201202026).

\section{References}

1. Demonty G, Bernard-Marty C, Puglisi F, et al: Progress and new standards of care in the management of HER-2 positive breast cancer. Eur J Cancer 43: 497-509, 2007.

2. Whenham N, D'Hondt V and Piccart MJ: HER2-positive breast cancer: from trastuzumab to innovatory anti-HER2 strategies. Clin Breast Cancer 8: 38-49, 2008.

3. Yarden Y, Baselga J and Miles D: Molecular approach to breast cancer treatment. Semin Oncol 31 (5 Suppl 10): S6-S13, 2004.

4. Zhang ZJ and Ma SL: miRNAs in breast cancer tumorigenesis (Review). Oncol Rep 27: 903-910, 2012.

5. Chia S, Norris B, Speers C, et al: Human epidermal growth factor receptor 2 overexpression as a prognostic factor in a large tissue microarray series of node-negative breast cancers. J Clin Oncol 26: 5697-5704, 2008.

6. Semiglazov VF and Semiglazov VV: Neoadjuvant systemic therapy in breast cancer. In: Neoadjuvant Chemotherapy: Current Applications in Clinical Practice. Bathe OF (ed). Intech, Rijeka, pp1-22, 2012.

7. Semiglazov VF and Semiglazov VV: Neoadjuvant (Preoperative) Therapy in Breast Cancer. 2013.

8. von Minckwitz G, Untch M, Blohmer JU, et al: Definition and impact of pathologic complete response on prognosis after neoadjuvant chemotherapy in various intrinsic breast cancer subtypes. J Clin Oncol 30: 1796-1804, 2012.

9. Coudert BP, Largillier R, Arnould L, et al: Multicenter phase II trial of neoadjuvant therapy with trastuzumab, docetaxel, and carboplatin for human epidermal growth factor receptor-2-overexpressing stage II or III breast cancer: results of the GETN (A)-1 trial. J Clin Oncol 25: 2678-2684, 2007.

10. Galdy S, Zenoni D, Galmozzi A, et al: Adjuvant concurrent docetaxel, epirubicin and cyclophosphamide chemotherapy in breast cancer: The TEC regimen. A retrospective analysis. J Cancer Res Ther 2: 40-47, 2014.

11. Hirano A, Shimizu T, Watanabe O, et al: Epirubicin and cyclophosphamide followed by docetaxel as primary systemic chemotherapy in locally advanced breast cancer. Anticancer Res 28: 4137-4142, 2008.

12. Yao X, Hosenpud J, Chitambar CR, et al: A phase II study of concurrent docetaxel, epirubicin and cyclophosphamide as a neoadjuvant chemotherapy regimen in patients with locally advanced breast cancer. J Cancer 3: 145-151, 2012.

13. Gianni L, Eiermann W, Semiglazov V, et al: Neoadjuvant chemotherapy with trastuzumab followed by adjuvant trastuzumab versus neoadjuvant chemotherapy alone, in patients with HER2-positive locally advanced breast cancer (the NOAH trial): a randomised controlled superiority trial with a parallel HER2-negative cohort. Lancet 375: 377-384, 2010.

14. Pegram M and Liao J: Trastuzumab treatment in multiple lines: current data and future directions. Clin Breast Cancer 12: 10-18, 2012.

15. Greene FL and Sobin LH: A worldwide approach to the TNM staging system: collaborative efforts of the AJCC and UICC. J Surg Oncol 99: 269-272, 2009.

16. Li Z, Luo Y, Gong Y, Liu Y, Qiu W and Tu J: Clinical features and molecular phenotypes of breast cancer in patients with type-2 diabetes mellitus. Asian Pac J Cancer Prev 12: 21832188, 2011.

17. Khabaz MN: Immunohistochemistry subtypes (ER/PR/HER) of breast cancer: where do we stand in the West of Saudi Arabia? Asian Pac J Cancer Prev 15: 8395-8400, 2014.

18. Allred DC, Harvey JM, Berardo M and Clark GM: Prognostic and predictive factors in breast cancer by immunohistochemical analysis. Modern Pathology 11: 155-168, 1998.

19. Almasri NM and Al Hamad M: Immunohistochemical evaluation of human epidermal growth factor receptor 2 and estrogen and progesterone receptors in breast carcinoma in Jordan. Breast Cancer Res 7: R598-R604, 2005.

20. Pauletti G, Godolphin W, Press MF and Slamon DJ: Detection and quantitation of HER-2/neu gene amplification in human breast cancer archival material using fluorescence in situ hybridization. Oncogene 13: 63-72, 1996. 
21. Hayward JL, Carbone PP, Heuson JC, et al: Assessment of response to therapy in advanced breast cancer: a project of the Programme on Clinical Oncology of the International Union Against Cancer, Geneva, Switzerland. European J Cancer 13: 89-94, 1977.

22. Huang D: TEC program neoadjuvant chemotherapy for locally advanced breast cancer observed. Hebei Med 18: 519-521, 2012 (In Chinese).

23. Herrada J, Iyer RB, Atkinson EN, Sneige N, Buzdar AU and Hortobagyi GN: Relative value of physical examination, mammography, and breast sonography in evaluating the size of the primary tumor and regional lymph node metastases in women receiving neoadjuvant chemotherapy for locally advanced breast carcinoma. Clin Cancer Res 3: 1565-1569, 1997.

24. Kaufmann M, Hortobagyi GN, Goldhirsch A, et al: Recommendations from an international expert panel on the use of neoadjuvant (primary) systemic treatment of operable breast cancer: an update. J Clin Oncol 24: 1940-1949, 2006.

25. Fu Q, Xia S, Li C and Yu S: Analysis of long-term cardiotoxicity of adjuvant trastuzumab therapy in 26 HER2-positive breast cancer patients. Chin J Clin Oncol 29: 1952-1955, 2012 (In Chinese)

26. Guo L, Hou X, Dilimina Y and Wang B: Expression of ER $\beta$, $\mathrm{ER} \alpha$ and Her-2 and distribution of molecular subtypes in Uygur and Han patients with breast cancer. Exp Ther Med 7: 1077-1082, 2014.

27. Untch M, Fasching PA, Konecny GE, et al: Pathologic complete response after neoadjuvant chemotherapy plus trastuzumab predicts favorable survival in human epidermal growth factor receptor 2-overexpressing breast cancer: results from the TECHNO trial of the AGO and GBG study groups. J Clin Oncol 29: 3351-3357, 2011.

28. Lazaridis G, Pentheroudakis G and Pavlidis N: Integrating trastuzumab in the neoadjuvant treatment of primary breast cancer: accumulating evidence of efficacy, synergy and safety. Crit Rev Oncol Hematol 66: 31-41, 2008.

29. Slamon D, Eiermann W, Robert N, et al; Breast Cancer International Research Group: Adjuvant trastuzumab in HER2-positive breast cancer. N Engl J Med 365: 1273-1283, 2011.
30. Esteva FJ, Valero V, Booser D, et al: Phase II study of weekly docetaxel and trastuzumab for patients with HER-2-overexpressing metastatic breast cancer. J Clin Oncol 20: 1800-1808, 2002.

31. Marty M,Cognetti F, Maraninchi D, et al: Randomized phase II trial of the efficacy and safety of trastuzumab combined with docetaxel in patients with human epidermal growth factor receptor 2-positive metastatic breast cancer administered as first-line treatment: the M77001 study group. J Clin Oncol 23: 4265-4274, 2005.

32. Montemurro F, Choa G, Faggiuolo R, et al: Safety and activity of docetaxel and trastuzumab in HER2 overexpressing metastatic breast cancer: a pilot phase II study. Am J Clin Oncol 26: 95-97, 2003.

33. Tedesco KL, Thor AD, Johnson DH, et al: Docetaxel combined with trastuzumab is an active regimen in HER-2 3+ overexpressing and fluorescent in situ hybridization-positive metastatic breast cancer: a multi-institutional phase II trial. J Clin Oncol 22: 1071-1077, 2004.

34. Untch M, Rezai M, Loibl S, et al: Neoadjuvant treatment with trastuzumab in HER2-positive breast cancer: results from the GeparQuattro Study. J Clin Oncol 28: 2024-2031, 2010.

35. Mauri D, Pavlidis N and Ioannidis JP: Neoadjuvant versus adjuvant systemic treatment in breast cancer: a meta-analysis. J Nat Cancer Inst 97: 188-194. 2005.

36. Wolmark N, Wang J, Mamounas E, Bryant J and Fisher B: Preoperative chemotherapy in patients with operable breast cancer: nine-year results from National Surgical Adjuvant Breast and Bowel Project B-18. J Natl Cancer Inst Monogr 30: 96-102. 2001.

37. Suter TM, Procter M, van Veldhuisen DJ, et al: Trastuzumab-associated cardiac adverse effects in the herceptin adjuvant trial. J Clin Oncol 25: 3859-3865. 2007.

38. Baselga J, Norton L, Albanell J, Kim YM and Mendelsohn J: Recombinant humanized anti-HER2 antibody (Herceptin) enhances the antitumor activity of paclitaxel and doxorubicin against HER2/neu overexpressing human breast cancer xenografts. Cancer Res 58: 2825-2831, 1998.

39. Palmer BV, Walsh GA, McKinna JA and Greening WP: Adjuvant chemotherapy for breast cancer: side effects and quality of life. Br Med J 281: 1594-1597, 1980. 